\title{
Phase Diagram of Colloidal Hard Superballs: from Cubes via Spheres to Octahedra
}

\author{
Ran Ni (倪再), , * Anjan Prasad Gantapara, ${ }^{1}$ Joost de Graaf, ${ }^{1}$ René van Roij, ${ }^{2}$ and Marjolein Dijkstra ${ }^{1}$, \\ ${ }^{1}$ Soft Condensed Matter, Utrecht University, Princetonplein 5, 3584 CC Utrecht, The Netherlands \\ ${ }^{2}$ Institute for Theoretical Physics, Utrecht University, \\ Leuvenlaan 4, 3504 CE Utrecht, The Netherlands
}

(Dated: August 29, 2018)

\begin{abstract}
The phase diagram of colloidal hard superballs, of which the shape interpolates between cubes and octahedra via spheres, is determined by free-energy calculations in Monte Carlo simulations. We discover not only a stable face-centered cubic (fcc) plastic crystal phase for near-spherical particles, but also a stable body-centered cubic (bcc) plastic crystal close to the octahedron shape. Moreover, coexistence of these two plastic crystals is observed with a substantial density gap. The plastic fcc and bcc crystals are, however, both unstable in the cube and octahedron limit, suggesting that the rounded corners of superballs play an important role in stablizing the rotator phases. In addition, we observe a two-step melting phenomenon for hard octahedra, in which the Minkowski crystal melts into a metastable bcc plastic crystal before melting into the fluid phase.

PACS numbers: 82.70.Dd, 64.70.pv, 64.75.-g, 64.75.Yz, 61.50.-f, 64.60.-i
\end{abstract}

Recent breakthroughs in particle synthesis have resulted in a spectacular variety of anisotropic nanoparticles such as cubes, octapods, tetrapods, octahedra, icecones, etc. [1]. A natural starting point to study the selfassembled structures of these colloidal building blocks is to view them as hard particles [1]. Not only can these hard-particle models be used to predict properties of suitable experimental systems, but such models also provide a stepping stone towards systems where soft interactions play a role [2, 3]. Moreover, the analysis of hard particles is of fundamental relevance and raises problems that influence fields as diverse as (soft) condensed matter [1, 46], mathematics [5, 7], and computer science [8]. In this light the concurrent boom in simulation studies of hard anisotropic particles is not surprising [5-7, $9-16]$.

The best-known hard-particle system consists of hard spheres, which freeze into close-packed hexagonal (cph) crystal structures [8], of which the ABC-stacked cph crystal, better known as the face-centered cubic (fcc) crystal phase, is thermodynamically stable [17]. Hard anisotropic particles can form liquid-crystalline equilibrium states if they are sufficiently rod- or disclike [12, 16], but particles with shapes that are close-to-spherical tend to order into plastic crystal phases, also known as rotator phases [14 16]. In fact, simple guidelines were recently proposed to predict the plastic- and liquid-crystal formation only on the basis of rotational symmetry and shape anisotropy of hard polyhedra [6]. In this Letter we will take a different approach, based on free-energy calculations, and address the question whether and to what extent rounding the corners and faces of polyhedral particles affects the phase behavior. Such curvature effects are of direct relevance to experimental systems, in which sterically and charged stabilised particles can often not be considered as perfectly flat-faced and sharp-edged [18]. For instance, recent experiments on nanocube assemblies show a continuous phase transformation between simple cubic and rhombohedral phases by increasing the ligand thickness and hence the particle sphericity [3].

In this Letter, we study a system of colloidal hard superballs in order to address these problems. A superball is defined by the inequality

$$
|x|^{2 q}+|y|^{2 q}+|z|^{2 q} \leq 1,
$$

where $x, y$ and $z$ are scaled Cartesian coordinates with $q$ the deformation parameter. The shape of the superball interpolates smoothly between two Platonic solids, namely the octahedron $(q=0.5)$ and the cube $(q=\infty)$ via the sphere $(q=1)$. By determining the phase diagram of these superballs as a function of $q$, we discovered a thermodynamically stable body-centered cubic (bcc) plastic crystal phase for octahedron-like superballs. To the best of our knowledge no plastic crystals other than cph structures have so far been observed for hard particles. Moreover, we find that bcc and fcc plastic crystal phases are unstable for hard octahedra and hard cubes, respectively. Therefore, rounded faces and edges may play an important role in stabilizing rotator phases, while flat faces tend to stabilize crystals.

Following Refs. [9, 19], we first calculate the closepacked structures for systems of hard superballs. We employ the algorithm of checking overlap described in Ref. [20]. For cube-like particles, it is found that at close packing there are so-called $C_{0}$ and $C_{1}$ crystal phases in accordance with Ref. [10]. When we perform NPT Monte Carlo simulations with variable box shape to determine the equation of state (EOS) of the crystal phase, our simulation results show that both the $C_{0}$ and the $C_{1}$ crystals deform with decreasing density. The lattice vectors for $C_{1}$ crystals are given by $\mathbf{e}_{1}=2^{1-1 / 2 q} \mathbf{i}+2^{1-1 / 2 q} \mathbf{j}$ $, \mathbf{e}_{2}=2^{1-1 / 2 q} \mathbf{i}+2^{1-1 / 2 q} \mathbf{k}, \mathbf{e}_{3}=2\left(s+2^{-1 / 2 q}\right) \mathbf{i}-2 s \mathbf{j}-$ $2 s \mathbf{k}$, where $\mathbf{i}, \mathbf{j}$ and $\mathbf{k}$ are the unit vectors along the axes of the particle, $s$ is the smallest positive root of the equation $\left(s+2^{-1 / 2 q}\right)^{2 q}+2 s^{2 q}-1=0$, and there is one parti- 


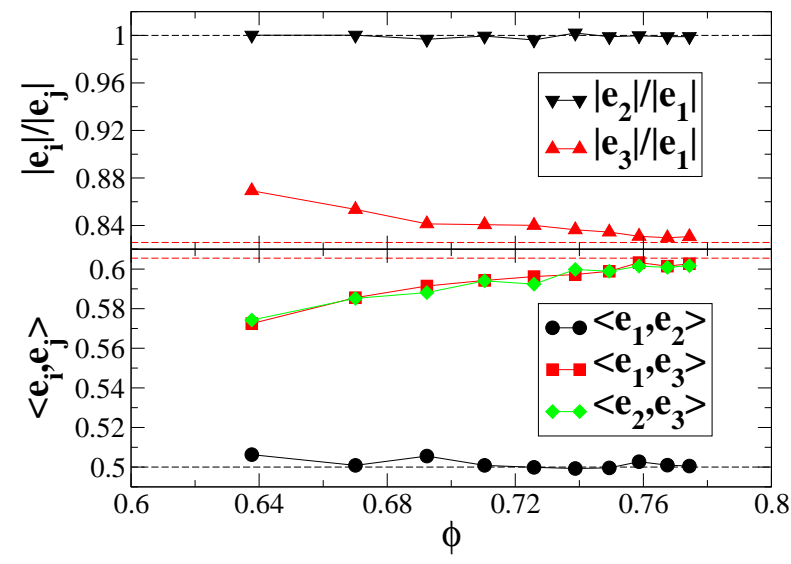

FIG. 1: (Color online) The deformation of the crystal unit cell with lattice vectors $\mathbf{e}_{i}$ as a function of packing fraction $\phi$ in a system of hard superballs with $q=2.5$. The dashed lines in the figures indicate the values for the $C_{1}$ crystal.

cle in the unit cell [10]. For instance, when $q=2.5$, one finds that $\left\langle\mathbf{e}_{1}, \mathbf{e}_{2}\right\rangle=0.5,\left\langle\mathbf{e}_{1}, \mathbf{e}_{3}\right\rangle=\left\langle\mathbf{e}_{3}, \mathbf{e}_{2}\right\rangle=0.60552$, $\left|\mathbf{e}_{2}\right| /\left|\mathbf{e}_{1}\right|=1$ and $\left|\mathbf{e}_{3}\right| /\left|\mathbf{e}_{1}\right|=0.825737$, where $\left\langle\mathbf{e}_{i}, \mathbf{e}_{j}\right\rangle$ is the cosine of angle between $\mathbf{e}_{i}$ and $\mathbf{e}_{j}$. The calculated angles and the length ratios between lattice vectors as a function of packing fraction $\phi$ for the cubelike particles with $q=2.5$ are shown in Fig. 1. We find that at packing fractions approaching close packing, the crystal remains in the $C_{1}$ phase. With decreasing packing fraction, the crystal lattice deforms towards an fcc structure: $\left\langle\mathbf{e}_{1}, \mathbf{e}_{2}\right\rangle=\left\langle\mathbf{e}_{1}, \mathbf{e}_{3}\right\rangle=\left\langle\mathbf{e}_{2}, \mathbf{e}_{3}\right\rangle=0.5$ and $\left|\mathbf{e}_{2}\right| /\left|\mathbf{e}_{1}\right|=\left|\mathbf{e}_{3}\right| /\left|\mathbf{e}_{1}\right|=1$. Moreover, when $1<q<3$, it is found that the deformed $C_{0}$ and deformed $C_{1}$ crystal melt into an fcc plastic crystal phase. By Einstein integration, we calculated the Helmholtz free energy as a function of packing fraction for both the fcc plastic crystal and the deformed $C_{1} / C_{0}$ crystal phases [21]. Combined with the free-energy calculations for the fluid phase done by Widom's particle insertion method, we obtain the phase boundaries in the phase diagram shown in Fig. 2. The part of the phase diagram for hard cube-like superballs roughly agrees with the empirical phase diagram by Batten et al. 111] At high packing fractions, there are stable deformed $C_{0}$ and $C_{1}$ phases. When $q>1.1509$, the close-packed structure is the $C_{1}$ crystal, whereas it is the $C_{0}$ crystal whenever $1<q<1.1509$ [10]. To determine the location of the transition from the deformed $C_{0}$ crystal to the deformed $C_{1}$ crystal, we performed two series of NPT MC simulations with increasing value of $q$ for the first series and decreasing $q$ for the second series of simulations at pressure $P^{*}=P v / k_{B} T \simeq 250$, with $k_{B}$ the Boltzmann constant, $T$ the temperature, and $v$ the volume of the particle [12]. The first series started from a $C_{0}$ crystal phase, while the second series of simu- lations started from a $C_{1}$ crystal phase. Our simulations show that the phase transition occurred around $q=1.09$ at packing fraction $\phi=0.736$ as shown by the asterix in Fig. 2. Moreover, for hard cubes $(q=\infty)$ the $C_{1}$ crystal is a simple cubic (sc) crystal. Although it was found that for hard cubes there is a significant amount of vacancies in the simple cubic crystal, it only shifts the phase boundary by $\sim 2 \%$ in packing fraction [13]. In our simulations, we did not observe any vacancies in the crystals of hard superballs with $q \leq 3$, we therefore assume that the possible presence of vacancies would not shift the phase boundary significantly.

The other part of the phase diagram concerns the octahedron-like superballs. For $0.79248<q<1$, we obtained a denser structure than the predicted $O_{0}$ lattice of Ref. 10]. For instance, after compressing the system to pressures around $P^{*}=10^{7}$ at $q=0.85$, we obtained a body-centered-tetragonal (bct) crystal with $\phi=0.7661$. This is denser than the $O_{0}$ crystal, which achieves $\phi=$ 0.7656 at $q=0.85$. Note however that these two crystals are very similar to each other, since $O_{0}$ is also a form of a bct lattice. The only difference is that the orientation of the particles in the $O_{0}$ crystal is the same as the symmetry of the axes in the crystal lattice, while in our bct crystal there is a small angle between these two orientations in the square plane of the crystal. Furthermore, for $q<0.79248$, we also found a crystal with denser packing than the predicted $O_{1}$ crystal in Refs. [10]. For $q=0.7$, we performed floppy-box MC simulations with several particles to compress the system to a high pressure state, i.e., $P^{*}=10^{7}$. We found a deformed bcc (dbcc) crystal shown in Fig. 2, which is an intermediate form between the bcc lattice and the Minkowski crystal [22]. The lattice vectors are $\mathbf{e}_{1}=0.912909 \mathbf{i}+0.912403 \mathbf{j}-0.912165 \mathbf{k}$, $\mathbf{e}_{2}=-0.271668 \mathbf{i}+1.80916 \mathbf{j}-0.288051 \mathbf{k}$ and $\mathbf{e}_{3}=$ $0.28834 \mathbf{i}-0.272001 \mathbf{j}-1.80882 \mathbf{k}$, where $\mathbf{i}, \mathbf{j}$ and $\mathbf{k}$ are the unit vectors along the axes of the particle. Our dbcc crystal is very close the predicted $O_{1}$ crystal, whose lattice vectors are $\mathbf{e}_{1}=0.912492 \mathbf{i}+0.912492 \mathbf{j}-0.912492 \mathbf{k}$, $\mathbf{e}_{2}=-0.2884 \mathbf{i}+1.80629 \mathbf{j}-0.2884 \mathbf{k}$, and $\mathbf{e}_{3}=0.2884 \mathbf{i}-$ $0.2884 \mathbf{j}-1.80629 \mathbf{k}$. However, it has a packing fraction of $\phi=0.832839$ which is denser than the predicted $O_{1}$ crystal with $\phi=0.824976$ in Refs. [10] by roughly $1 \%$. In Ref. [10], the $O_{0}$ and $O_{1}$ phases are found to switch at $q=0.79248$. We also observed that the bct and dbcc crystals both transform into the bcc phase at $q=0.79248$.

As shown in Fig. 2, when the shape of the superballs is close to spherical, i.e., $0.7<q<3$, there is always a stable fcc plastic crystal phase. Surprisingly, when the shape of superballs is octahedron-like, we find a stable bcc plastic crystal phase. Moreover, around $q=0.8$ we even find a fairly broad two-phase regime where a lowdensity fcc plastic crystal coexists with a high-density bcc plastic crystal phase. In order to quantify the orientational order in the bcc plastic crystal, we calculate the 


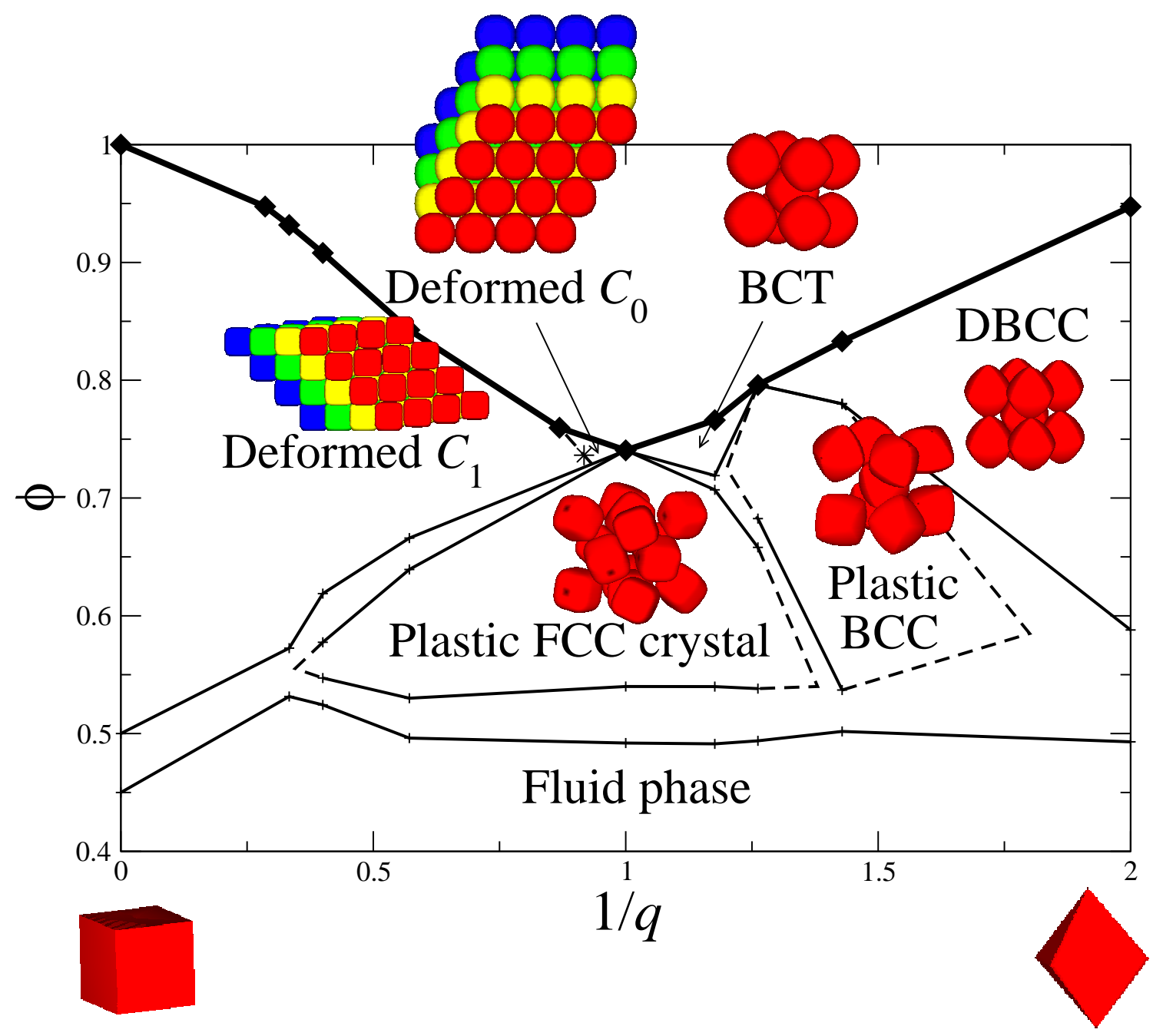

FIG. 2: (Color online) Phase diagram for hard superballs in the $\phi$ (packing fraction) versus $1 / q$ representation where $q$ is the deformation parameter. Here the $C_{1}$ and $C_{0}$ crystals are defined in Ref. [10], where the particles of the same color are in the same layer of stacking. The solid diamonds indicate the close packing, and the locations of triple points are determined by extrapolation as shown by the dashed lines. The phase boundaries for hard cubes are taken from Ref. [13].

cubatic order parameter $S_{4}$ given by [11]

$$
S_{4}=\max _{\mathbf{n}}\left\{\frac{1}{14 N} \sum_{i, j}\left(35\left|\mathbf{u}_{i j} \cdot \mathbf{n}\right|^{4}-30\left|\mathbf{u}_{i j} \cdot \mathbf{n}\right|^{2}+3\right)\right\},
$$

where $\mathbf{u}_{i j}$ is the unit vector of the $j$-th axis of particle $i$, $N$ the number of particles, and $\mathbf{n}$ is a unit vector. The cubatic order parameter $S_{4}$ is shown in Fig. 3 as a function of packing fraction for a system of superballs with $q=0.7$. We observe that $\left\langle S_{4}\right\rangle \simeq 0.2$ at low packing fractions, which means that there is a very weak orientational order in the system [20]. With increasing packing fraction, the cubatic order parameter increases monotonically to around 0.65 at a packing fraction of 0.7 , which is indicative of a medium-ranged orientationally ordered system. This suggests that the entropic repulsion due to the rotation of the octahedron-like superballs stablizes the bcc lattice.

Due to the numerical instability in the overlap algorithm, we are not able to investigate systems of superballs with $q<0.7$ 23]. However, we can use the separating axis theorem [6] to simulate hard superballs with $q=0.5$, i.e., perfect octahedra. When we compressed the system from a fluid phase, we did not observe the spontaneous formation of a crystal phase in our simulation box within our simulation time. When we expand the Minkowski crystal, which is the close-packed structure of octahedra, in NPT MC simulations by decreasing the pressure, the system melts into a bcc plastic crystal phase as shown in Fig. 4. We also calculated the free energy for these three phases to determine the phase boundaries. To exclude finite-size effects in the free-energy calculation of crystal phases, we performed Einstein integration 


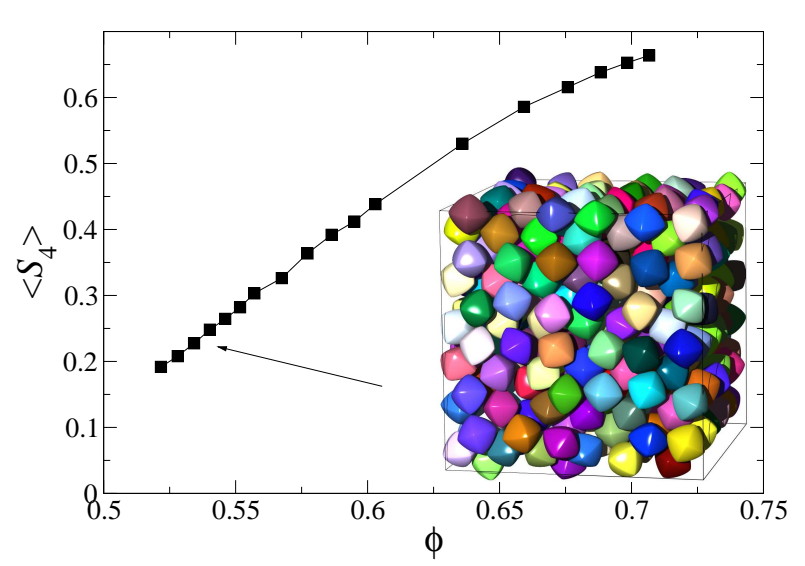

FIG. 3: (Color online) Cubatic order parameter $S_{4}$ as a function of packing fraction $\phi$ for a bcc plastic crystal phase of hard superballs with $q=0.7$. The inset shows a typical configuration of a bcc plastic crystal of hard superballs with $q=0.7$ at $\phi=0.54$.

for systems of $N=1024,1458$, and 2000 particles, and applied a finite-size correction [21]. We confirmed the errors in the free-energy calculations to be on the order of $10^{-3} k_{B} T$ per particle. The calculated free-energy densities for the three phases are shown in Fig. 4. Employing a common tangent construction, we found that there is only phase coexistence between a fluid phase and a Minkowski crystal phase, while the bcc plastic crystal phase is metastable. However, the free-energy differences between the fluid and the plastic crystal phase at the bulk coexistence pressure is very small, i.e., $\sim 10^{-2} k_{B} T$ per particle. Moreover, the Minkowski crystal melts into a bcc plastic crystal before melting into the fluid phase. Our results thus show that the rounded corners of octahedra play an important role in stablizing the bcc plastic crystal phase which is a new plastic crystal phase for systems of hard particles.

In conclusion, using free-energy calculations we have determined the full phase diagram of hard superballs with shapes interpolating between cubes and octahedra, i.e., $0.5 \leq q<\infty$. In systems of cube-like superballs $(q>1)$, we find a stable deformed $C_{1}$ phase at high packing fraction, except close to the sphere-limit $(q=1)$ where a deformed $C_{0}$ crystal is stable. For $q<3$ the crystal phase melts into an fcc plastic crystal before melting into a fluid phase of cubelike superballs. In systems of octahedron-like superballs $(0.5<q<1)$, we find a stable bct or a deformed bcc crystal phase upon approaching close packing, with a crossover at $q=0.79248$. Moreover, a stable fcc plastic crystal appears at intermediate densities for $0.7<q \leq 1$. Interestingly, for $q<0.85$, we find a novel stable bcc plastic crystal phase, which can even coexist with the fcc plastic crystal phase at around

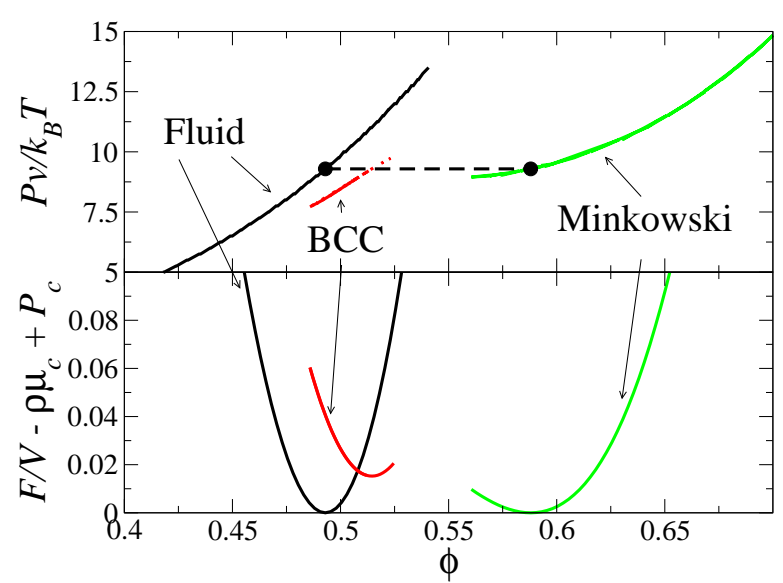

FIG. 4: (Color online) A part of the equation of state for hard octahedra. The pressure $P v / k_{B} T$ and free-energy density $F / V-\rho \mu_{c}+P_{c}$ as a function of packing fraction $\phi$. Here $v$ is the volume of the particle; $F$ and $V$ are the Helmholtz free energy and the volume of the system (in units of particle volume) respectively; $\mu_{c}$ and $P_{c}$ are the chemical potential and pressure at bulk coexistence respectively with $\rho$ the number density of the particles. The solid lines in the EOS for the Minkowski and the bcc plastic crystal phases are obtained by melting the close-packed Minkowski crystal in floppy box NPT MC simulations, and the dotted line for the bcc plastic crystal is obtained by compressing the crystal in cubic box NPT MC simulations. The black points and dashed line show the coexistence between the fluid phase and the Minkowski crystal phase.

$q=0.8$. More surprisingly, the bcc and fcc rotator phases are unstable for the flat-faced and sharp-edged hard octahedra and hard cubes, respectively, which suggests that the rounded corners of superballs may play an important role in stabilizing rotator phases. It is tempting to argue that entropic directional forces 24] that tend to align sufficiently large flat faces of polyhedral-shaped particles destabilize rotator phases in favor of crystals. We stress here that rounded corners are not a necessary condition for stable rotator phases since almost spherical polyhedral particles have been shown to form rotator phases as well [6]. Finally, we also observed a two-step melting phenomenon in the system of hard octahedra, such that the Minkowski crystal melts into a metastable bcc plastic crystal before melting into the fluid phase. Nanoparticle self-assembly is therefore surprisingly sensitive to particle curvature.

We thank F. Smallenburg for fruitful discussions and Dr. D. Ashton for making the snapshot in Fig. 3, We acknowledge the financial support from a NWO-VICI grant and Utrecht University High Potential Programme. 
* Electronic address: r.ni@uu.nl

† Electronic address: m.dijkstra1@uu.nl

[1] S. C. Glotzer and M. J. Solomon, Nature Mater. 6, 557 (2007).

[2] A. Yethiraj and A. van Blaaderen, Nature (London) 421, 513 (2003).

[3] Y. Zhang, F. Lu, D. van der Lelie, and O. Gang, Phys. Rev. Lett. 107, 135701 (2011).

[4] A.-P. Hynninen, J. H. J. Thijssen, E. C. M. Vermolen, M. Dijkstra, and A. van Blaaderen, Nature Mater. 6, 202 (2007).

[5] A. Haji-Akbari, M. Engel, A. S. Keys, X. Zheng, R. G. Petschek, P. Palffy-Muhoray, and S. C. Glotzer, Nature (London) 462, 773 (2009).

[6] U. Agarwal and F. Escobedo, Nature Mater. 10, 230 (2011).

[7] S. Torquato and Y. Jiao, Nature (London) 460, 876 (2009).

[8] T. C. Hales and S. P. Ferguson, Discrete Comput. Geom. 36, 5 (2006).

[9] J. de Graaf, R. van Roij, and M. Dijkstra, Phys. Rev. Lett. 107, 155501 (2011).

[10] Y. Jiao, F. H. Stillinger, and S. Torquato, Phys. Rev. E 79, 041309 (2009); ibid, Phys. Rev. E 84, 069902 (2011).

[11] R. D. Batten, F. H. Stillinger, and S. Torquato, Phys. Rev. E 81, 061105 (2010).
[12] M. Marechal, A. Cuetos, B. Martínez-Haya, and M. Dijkstra, J. Chem. Phys. 134, 094501 (2011).

[13] F. Smallenburg, L. Filion, M. Marechal, and M. Dijkstra, submitted (2011), arXiv:1111.3466v2 [cond-mat.soft].

[14] C. Vega and P. A. Monson, J. Chem. Phys. 107, 2696 (1997).

[15] M. Marechal and M. Dijkstra, Phys. Rev. E 77, 061405 (2008).

[16] P. Bolhuis and D. Frenkel, J. Chem. Phys. 106, 666 (1997).

[17] P. G. Bolhuis, D. Frenkel, S.-C. Mau, and D. A. Huse, Nature 388, 235 (1997).

[18] L. Rossi, S. Sacanna, W. T. M. Irvine, P. M. Chaikin, D. J. Pine, and A. P. Philipse, Soft Matter 7, 4139 (2011).

[19] L. Filion, M. Marechal, B. van Oorschot, D. Pelt, F. Smallenburg, and M. Dijkstra, Phys. Rev. Lett. 103, 188302 (2009).

[20] See Supplemental Material at http://unknown for technical details and the movie of plastic bcc crystal formed by superballs with $q=0.7$ at packing fraction 0.54 .

[21] D. Frenkel and B. Smit, Understanding Molecular Simulation: From Algorithms to Applications (Academic Press, 2002).

[22] H. Minkowski, Königliche Gesellschaft der Wissenschaften zu Göttingen pp. 311-355 (1904).

[23] A. Donev, Ph.D. thesis, Princeton University (2006).

[24] P. F. Damasceno, M. Engel, and S. C. Glotzer (2011), arXiv:1109.1323v2 [cond-mat.soft]. 